\title{
Patient-related factors, antibiotic prescribing and antimicrobial resistance of the commensal Staphylococcus aureus and Streptococcus pneumoniae in a healthy population - Hungarian results of the APRES study
}

László Róbert Kolozsvári ${ }^{*}$ (D) József Kónya² ${ }^{2}$ John Paget ${ }^{3}$, Francois G. Schellevis ${ }^{3,6}$, János Sándor ${ }^{4}$, Gergő József Szőllősi ${ }^{4}$, Szilvia Harsányi ${ }^{5}$, Zoltán Jancsó ${ }^{1}$ and Imre Rurik ${ }^{1}$

\begin{abstract}
Background: Antimicrobial resistance (AMR) is an increasing public health problem worldwide. We studied some patient-related factors that might influence the antimicrobial resistance. and whether the volume of antibiotic prescribing of the primary care physicians correlate with the antibiotic resistance rates of commensal nasal Staphylococcus aureus and Streptococcus pneumoniae.

Methods: The socio-demographic questionnaires, the antibiotic prescription and resistance data of commensal nasal S. aureus and S. pneumoniae were collected in the 20 participating Hungarian practices of the APRES study. Multivariate logistic regression analyses were performed on the patient-related data and the antimicrobial resistance of the S. aureus and S. pneumoniae on individual, patient level.

Ecological analyses were performed with Spearman's rank correlations at practice level, the analyses were performed in the whole sample (all practices) and in the cohorts of primary care practices taking care of adults (adult practices) or children (paediatric practices).

Results: According to the multivariate model, age of the patients significantly influenced the antimicrobial resistance of the $S$. aureus $(\mathrm{OR}=0.42, p=0.004)$ and $S$. pneumoniae $(\mathrm{OR}=0.89, p<0.001)$. Living with children significantly increased the AMR of the $S$. pneumoniae ( $O R=1.23, p=0.019)$. In the cohorts of adult or paediatric practices, neither the age nor other variables influenced the AMR of the $S$. aureus and $S$. pneumoniae. At practice level, the prescribed volume of penicillins significantly correlated with the resistance rates of the $S$. aureus isolates to penicillin ( $r h o=0.57, p=0.008$ ). The volume of prescribed macrolides, lincosamides showed positive significant correlations with the S. pneumoniae resistance rates to clarithromycin and/or clindamycin in all practices (rho $=0.76, p=0.001$ ) and in the adult practices (rho $=0.63, p=0.021$ ).

(Continued on next page)
\end{abstract}

\footnotetext{
* Correspondence: kolozsvari.laszlo@sph.unideb.hu

${ }^{1}$ Department of Family and Occupational Medicine, Faculty of Public Health,

University of Debrecen, Debrecen, Hungary

Full list of author information is available at the end of the article
}

(c) The Author(s). 2019 Open Access This article is distributed under the terms of the Creative Commons Attribution 4.0 International License (http://creativecommons.org/licenses/by/4.0/), which permits unrestricted use, distribution, and reproduction in any medium, provided you give appropriate credit to the original author(s) and the source, provide a link to the Creative Commons license, and indicate if changes were made. The Creative Commons Public Domain Dedication waiver (http://creativecommons.org/publicdomain/zero/1.0/) applies to the data made available in this article, unless otherwise stated. 
(Continued from previous page)

Conclusions: The age is an important influencing factor of antimicrobial resistance. The results also suggest that there may be an association between the antibiotic prescribing of the primary care providers and the antibiotic resistance of the commensal S. aureus and S. pneumoniae. The role of the primary care physicians in the appropriate antibiotic prescribing is very important to avoid the antibiotic resistance.

Keywords: Antimicrobial resistance, Antibacterial resistance, Commensal, Healthy population, Patient-related factors, Staphylococcus aureus, Streptococcus pneumoniae

\section{Background}

Antimicrobial resistance (AMR), in particular antibacterial resistance $(A B R)$ is an increasing public health problem worldwide [1]. The available antibiotics might become ineffective against infections due to antimicrobial resistance, although there is a continuous research and development of new antibiotics. There are only a limited number of new antibiotics available in primary care [2], so avoiding resistance with all the possible means is very important.

About $90 \%$ of the antibiotics are prescribed by primary care providers $[3,4]$.

It has been shown that the overuse of antibiotics increases the risk of side effects, raises costs and is associated with increased AMR levels [5]. The resistance rates of the bacteria are influenced by prescribers' prescribing habits and by health policies as well [6].

Higher prescribing rates of antibiotics may increase the resistance in individuals, on the community level and in the whole society [7-9]. The results of the analysis of the outpatient antibiotic use and resistance in 26 countries in Europe showed significant correlation between increased consumption of antibiotics and the resistance of the microorganisms at national level [3].

Antibiotic use is a major contributing factor to the development of resistant bacteria, but many other factors may contribute: e.g. patients' age, movement and migration, socio-economic factors, dose and duration of the antibiotic treatment, gene transfer and clonal spread [10]. Human beings carry a very large number of commensal organisms which probably provide a significant but underestimated reservoir of resistance genes [11]. How these influence the development of resistance in pathogenic organisms is as yet uncertain [12].

The APRES study (The Appropriateness of PREScribing antibiotics in primary health care in Europe with respect to antibiotic resistance) was designed to establish resistance patterns of Staphylococcus aureus and Streptococcus pneumoniae in the nasal flora in community-dwelling individuals and to collect data about the antibiotic prescribing patterns of primary care providers (family physicians/general practitioners, primary care paediatricians) in nine European countries, the project was closed in 2014 [9, 13, 14].
The results of the APRES study in other countries suggest that age is one of the major determining factors of the prevalence of $S$. aureus carriership, but there may be also other factors that influence the nasal carriage $[15,16]$.

Another previously published result of the APRES study suggests that higher resistance rates of $S$. aureus are associated with younger age, but also the primary care physicians' antibiotic prescribing behaviour may influence the odds of the carriage of the resistant nasal commensal $S$. aureus and there is a need for further investigations on a national level [17].

The antibiotics are prescription only medicines, that can only be prescribed by doctors in Hungary. The national total antibiotic consumption was relatively constant between 1996 and 2003, but there were large differences between the community antibiotic consumption of the regions and between counties [18, 19]. Other previously published Hungarian studies about the ambulatory antibiotic consumption established a constant quantity $(18.0 \pm 1.8 \mathrm{DDD} / 1000$ inhabitants/day) between the years 2006-2010 [20].

Primary care practices are usually the first point of access to health care in Hungary. There are about 6500 primary care (PC) practices for a population of approximately 10 million in Hungary. There are three main types of $\mathrm{PC}$ practices in Hungary: adult practices $(\mathrm{n} \approx 3500)$ treating adults only, paediatric practices $(\mathrm{n} \approx 1500)$ with primary care paediatricians mostly in bigger towns and cities, caring of children under 18 years only, and mixed practices $(n \approx 1500)$ treating people of all ages (mainly established in smaller settlements, villages) [21].

The antimicrobial resistance at younger patients may be higher and the primary care providers antibiotic prescribing may influence the resistance rates of the commensal S. aureus and S. pneumoniae, we found no previous publications that investigated the antimicrobial resistance of two commensal bacteria at individual and at primary care practice level.

The aim of this study was to investigate, based on the Hungarian data of the APRES Study:

1. The patient-related factors that may influence the antimicrobial resistance of the commensal S. aureus and $S$. pneumoniae at patient level. 
2. Whether the volume of antibiotic prescribing of primary care providers correlate with the antibiotic resistance rates at practice level.

\section{Methods}

The APRES study design was published in previous articles in detail $[13,14]$, we therefore mainly focus on the methodology related to our study.

\section{Patients and practices}

Socio-demographic data of the participating patients (eg. age, gender, living with children, number of GP visits during the previous year etc.) and data of the practices (e.g. number of registered patients, practice type etc.) were collected with questionnaires.

The Hungarian part of the study was conducted in 20 primary care practices, patients were selected in all 3 types of Hungarian primary care practices (13 adult, 5 paediatric and 2 mixed practices) between November 2010 and March 2011.

The number of participating patients and primary care practices were calculated and established by an international group of experts and included in the APRES Study protocol $[13,14]$.

The participating practices were located in 5 counties (Borsod-Abaúj-Zemplén, Csongrád, Hajdú-Bihar, Heves, Szabolcs-Szatmár-Bereg) and in the capital (Budapest). The practice locations represented the main types of settlements across Hungary: the capital (1), larger cities (10), towns (5), large village (1) and villages (3 practices).

\section{Bacterial specimens}

Bacterial specimens were collected from a random sample of persons over 4 years of age, who attended the primary care practices and had no symptoms of an infectious disease. Other exclusion criteria were antibiotic use or hospitalisation in past 3 months, severe health problems (terminal illness, immunocompromised).

Nasal swabs were obtained from the individuals according to a routine protocol. The specimens were immediately inserted into a container tube, with charcoal transport medium and transferred into the Department of Medical Microbiology at the University of Debrecen within $48 \mathrm{~h}$. Colonies with characteristic Staphylococcus morphology were identified as $S$. aureus based on positive coagulase test and colonies of $S$. pneumoniae were identified on the basis of colony morphology and resistance to optochin by the national laboratory, then transferred and tested for resistance against antibiotics in one central laboratory (Maastricht University). Both $S$. aureus and $S$. pneumoniae isolates were stored in frozen skimmed milk under liquid nitrogen until transporting them to Maastricht University where antibiotic resistance were established quantitatively with a broth dilution method in micro-titre plates according to EUCAST recommendations [13, 22]. The $S$. aureus and S. pneumoniae isolates were tested for the following antimicrobials: penicillins with and without beta-lactamase inhibitors, cephalosporines, macrolides, tetracyclines, quinolones, trimethoprim-sulfamethoxazole and rifampicin; for $S$. aureus also aminoglycosides, fusidic acid and mupirocin were tested $[13,14]$.

Resistance was considered present if there was resistance to one or more antibiotic $(R \geq 1)$. Furthermore, we considered the resistance rates to penicillin (J01C) and azithromycin and/or clindamycin (J01F) specifically. The resistance rates were also calculated in cohorts of practices (all practices, adult practices, paediatric practice).

\section{Antibiotic prescribing}

The antibiotic prescribing data were collected from the 20 primary care physicians' computer databases. All individual antibiotic prescriptions were classified by the active substance according to anatomic therapeutic classification (ATC), DDD (Defined Daily Dose), DPP (dose per package) and number of packages. In our study, the antibiotic prescribing of the primary care practices was expressed in DDD/1000 active patients/day, according to the WHO ATC/DDD methodology [23]. The antibiotic exposure was measured with the DDDs (not the number of packages), because this may be more appropriate for international and national comparison and to evaluate the correlation between the use of antibiotics and antimicrobial resistance $[4,24,25]$. There are opinions, that the main effect of the prescribed antibiotics to the resistance rates may last for up to one year, so we considered the prescribing data the year preceding the nasal sampling, in $2010[8,17,26]$.

\section{Statistical analysis}

Multivariate logistic regression models were performed on the patient-related data and the antimicrobial resistance (isolates that were resistant to at least one antibiotics, $\mathrm{R} \geq 1$ ) of the $S$. aureus and $S$. pneumoniae on individual, patient level. The association between the $S$. aureus and S. pneumoniae antimicrobial resistance (outcome/dependent variables) and the patient- related factors (explanatory/independent variables) were analysed.

The patient-related factors (independent variables) were patients' age (years), gender (female/male), job (working with livestock and/or in nursery and/or in healthcare), living with children (yes/no), number of GP visits during the previous year (1-4 visits/none, $5+$ visits/none). The analyses were performed in the whole sample (all practices) and in the cohorts of practices taking care of adult persons (adult practices) or children only (paediatric practices).

Due to the data collection methods and anonymous patient data, the antimicrobial resistance and individual antibiotic prescribing data could not be linked at individual 
level, this was one the reasons why we investigated the correlation between the antibiotic prescribing and resistance rates at practice level. The resistance rates were also calculated at practice level. We presumed that the most commonly prescribed antibiotics groups in primary care are the penicillins (Beta-lactam antibacterials, penicillins J01C) and macrolides (Macrolides, lincosamides and streptogramins J01F). Furthermore, we considered the resistance rates to penicillin (J01C) and azithromycin/clarithromycin and/or clindamycin (J01F) specifically. The associations between the usage of antibiotics (DDD/1000 patients/day) and the resistance rates were analysed by Spearman's rank correlation. The correlations were analysed in the whole sample (all practices) and we also calculated the correlation in the cohorts of adult practices and paediatric practices.

The statistical analyses were performed with STATA 11.1 software (Statacorp LP. College Station, TX, USA).

\section{Results}

\section{Patients and practices}

There were 4017 questionnaires collected from the participating patients. The age distribution of the participants was $4-19$ years: $28.2 \%, 20-64$ years: $50.4 \%$, and above 65 years: $21.3 \%$. Their gender distribution was $44.5 \%$ male $(n=1713), 55.5 \%$ female $(n=2137)$.

The average number of registered patients was 1905 persons per practice (range between 724 and 3308). The adult practices had 2219 registered patients on average, the mixed practices 1719 and the paediatric practices 1163 patients on their list in year 2010, respectively.

The number of active patients (patients who attended the primary care surgery at least once in 2010) was used as denominator in our ecological statistical analyses. The average number of active patients was 1534 persons per practice (range between 658 and 1976). On average, there were 1758 active patients in the adult, 1227 in the mixed practices and 1057 in the paediatric practices seen in year 2010, respectively.

\section{Bacterial specimens}

There were 4017 nasal swabs collected, the number of the incorrect sampling was 150 (absence of bacteria, including normal microbiota), the number of mismatched samples were 17 (absence of patient background information or laboratory data). 3850 swabs were tested in the laboratory, including 2471 swabs from the adult and 984 in the paediatric practices.

The prevalence of carrying commensal $S$. aureus was $14.1 \%$ in all practices $(n=541), 12.8 \%$ in adult practices $(n=317)$ and in $17.6 \%$ paediatric practices $(n=173)$. The prevalence of the S. pneumoniae was $4.1 \%$ in all practices $(n=159), 1.1 \%$ in adult practices $(n=28)$ and $12.1 \%$ in paediatric practices $(n=119)$.
The number and percentage of resistant isolates are shown in Table 1.

According to our descriptive statistics, the prevalence of antimicrobial resistance (resistance to one or more antibiotics $(\mathrm{R} \geq 1)$ and resistance against penicillins (J01C) and macrolides/lincosamides (J01F) of the S. aureus and S. pneumoniae seemed to be lower in the cohorts of adult practices and higher in the paediatric practices, comparing to the cohorts of all practices.

\section{Antibiotic prescribing}

There were 24,223 antibiotic prescriptions issued by the participating primary care providers in 2010 . The average volume of all prescribed antibiotics was 15.86 DDD/ 1000 active patients/day per practice, the most commonly prescribed antibiotics were the beta-lactam antibacterials, penicillins (ATC3 level J01C; 8.61 DDD/1000 active patients/day per practice), the macrolides/lincosamides (J01F; 3.47), the other beta-lactam antibacterials (J01D; 1.37); the quinolone antibacterials (J01 M; 1.17), the tetracyclines (J01A; 0.51), the sulphonamides and trimethoprim (J01E; 0.42), and the other antibacterials (J01X; 0.98).

The mean volume of the prescribed penicillins (J01C) was $8.86 \pm 1.8($ mean \pm SD) DDD per 1000 active patients/day in all practices, $4.96 \pm 2.74$ DDD per 1000 active patients/day in the adult practices. The mean volume of the prescribed macrolides/lincosamides (J01F) was $3.43 \pm 2.39$ DDD per 1000 active patients/day in all practices, $2.79 \pm 1.17$ DDD per 1000 active patients/day in the adult practices.

Patient-related factors and antimicrobial resistance of the S. aureus and S. pneumoniae

The age of the patients significantly influenced the resistance of the S. aureus and S. pneumoniae. The odds having antimicrobial resistance decreased in the group of the patients over 18 years of age $\left(\mathrm{OR}_{\mathrm{S} \text {.aureus }}=0.42, p=0.004\right.$; $\left.\mathrm{OR}_{\text {S.pneumoniae }}=0.89, \quad p<0.001\right)$. Living with children significantly increased $(\mathrm{OR}=1.23, p=0.019)$ the AMR of the S. pneumoniae.

Table 1 The resistance of S. aureus and S. pneumoniae in all practices, adult practices and paediatric practices

\begin{tabular}{llll}
\hline Resistance & All practices & Adult practices & Paediatric practices \\
\hline S. aureus & $n=541$ & $n=317$ & $n=173$ \\
$R \geq 1$ & $434(80.2 \%)$ & $237(74.8 \%)$ & $151(87.3 \%)$ \\
J01C & $410(75.8 \%)$ & $221(69.7 \%)$ & $145(83.8 \%)$ \\
J01F & $65(12.0 \%)$ & $32(10.1 \%)$ & $28(16.2 \%)$ \\
S. pneumoniae & $n=159$ & $n=28$ & $n=119$ \\
R $\geq 1$ & $112(70.4 \%)$ & $10(35.7 \%)$ & $95(79.8 \%)$ \\
J01C & $30(18.9 \%)$ & $3(10.7 \%)$ & $25(21.0 \%)$ \\
J01F & $18(11.3 \%)$ & $2(7.1 \%)$ & $15(12.6 \%)$ \\
\hline
\end{tabular}

$[R \geq 1$, resistance to one or more antibiotics; J01C resistance to penicillins; J01F resistance to macrolides/lincosamides] 
It appears that in the cohorts of adult and paediatric practices the age-related or other variables did not influence the AMR of S. aureus and S. pneumoniae (Table 2).

\section{The correlation between antibiotic prescribing and resistance rates}

The volume of prescribed penicillins (J01C) correlated significantly with the resistance rates of $S$. aureus isolates to penicillin, when including the data from all practices in the analyses (rho $=0.57, p=0.008$ ), this means a moderate positive correlation, but not correlated when considering the adult (rho $=0.30, p=0.319$ ) or paediatric (rho $=0.54, p=0.284$ ) practices only. There was no significant correlation between the volume of prescribed macrolides, lincosamides (J01F) and the S. aureus resistance rates to azithromycin and/or clindamycin (Table 3).

The volume of prescribed macrolides, lincosamides (J01F) showed strong positive significant correlation with the S. pneumoniae resistance rates to clarithromycin and/ or clindamycin in all practices (rho $=0.76, p<0.001)$ and in the adult practices (rho $=0.63, p=0.021$ ), but no correlation in the paediatric practices (rho $=0.52, p=0.191$ ). The correlation between the volume of prescribed penicillins (J01C) with the resistance rates of S. pneumoniae was not statistically significant (Table 4.)

\section{Discussion}

The age of the patients may be an important factor regarding antimicrobial resistance. However, when we investigated the cohorts of both adult and paediatric practices separately, there was no association between the age and resistance. Living with children in a same household is likely to increase the risk of having resistant S. pneumoniae isolates. Childhood seems to be a more important influencing factor than the age only, as it could be concluded from the previous publications $[14,16,17]$. The APRES Study, that studied apparently healthy population revealed reduced prevalence of both methicillin-sensitive and methicillin-resistant $S$. aureus isolates with older age, but the prevalence and resistance

Table 2 Multivariate logistic regression models on the patient-related variables and antimicrobial resistance of the S. aureus and S. pneumoniae

\begin{tabular}{|c|c|c|c|c|c|c|}
\hline \multirow[t]{3}{*}{ Variables } & \multicolumn{6}{|c|}{ S. aureus resistance } \\
\hline & \multicolumn{2}{|l|}{ All practices } & \multicolumn{2}{|c|}{ Adult practices } & \multicolumn{2}{|c|}{ Paediatric practices } \\
\hline & Odds Ratio & $p$-value & Odds Ratio & $p$-value & Odds Ratio & $p$-value \\
\hline \multicolumn{7}{|l|}{ Patient variables } \\
\hline Age groups (over 18 years/under 18 years) & 0.42 & 0.004 & 0.64 & 0.693 & 0.44 & 0.269 \\
\hline Gender (male/female) & 0.79 & 0.322 & 0.92 & 0.765 & 0.42 & 0.083 \\
\hline Job - Livestock (yes/no) & 0.91 & 0.970 & 1.01 & 0.980 & N/A & N/A \\
\hline Job - Nursery (yes/no) & 0.55 & 0.329 & 0.89 & 0.967 & N/A & N/A \\
\hline Job - Healthcare (yes/no) & 0.90 & 0.903 & 0.95 & 0.952 & N/A & N/A \\
\hline Living with children (yes/no) & 0.79 & 0.537 & 0.67 & 0.428 & 1.12 & 0.859 \\
\hline \multicolumn{7}{|l|}{ Number of GP visits } \\
\hline $1-4$ visits/none & 0.60 & 0.528 & 0.77 & 0.757 & 0.11 & 0.969 \\
\hline $5+$ visits/none & 0.90 & 0.898 & 1.18 & 0.859 & 0.10 & 0.971 \\
\hline \multirow[t]{3}{*}{ Variables } & \multicolumn{6}{|c|}{ S. pneumoniae resistance } \\
\hline & \multicolumn{2}{|l|}{ All practices } & \multicolumn{2}{|c|}{ Adult practices } & \multicolumn{2}{|c|}{ Paediatric practices } \\
\hline & Odds Ratio & $p$-value & Odds Ratio & $p$-value & Odds Ratio & $p$-value \\
\hline \multicolumn{7}{|l|}{ Patient variables } \\
\hline Age groups (over 18 years/under 18 years) & 0.89 & $<0.001$ & 1.00 & 0.842 & 0.79 & 0.785 \\
\hline Gender (male/female) & 1.45 & 0.425 & 1.87 & 0.503 & 1.27 & 0.646 \\
\hline Job - Livestock (yes/no) & 1.23 & 0.873 & 3.59 & 0.430 & N/A & N/A \\
\hline Job - Nursery (yes/no) & N/A & N/A & N/A & $\mathrm{N} / \mathrm{A}$ & N/A & N/A \\
\hline Job - Healthcare (yes/no) & 3.71 & 0.324 & 3.07 & 0.206 & N/A & N/A \\
\hline Living with children (yes/no) & 1.23 & 0.019 & 0.29 & 0.409 & 0.34 & 0.085 \\
\hline \multicolumn{7}{|l|}{ Number of GP visits } \\
\hline $1-4$ visits/none & 0.98 & 0.988 & 1.37 & 0.962 & 1.08 & 0.933 \\
\hline $5+$ visits/none & 0.98 & 0.925 & 0.93 & 0.409 & 1.03 & 0.978 \\
\hline
\end{tabular}

[significant results marked in bold] 
Table 3 Correlations between volume of prescribed penicillins and macrolides/lincosamides and resistance rates of S. aureus (\%) in all practices, adult and paediatric practices

\begin{tabular}{|c|c|c|c|}
\hline \multicolumn{4}{|l|}{ All practices } \\
\hline Prescription of & S. aureus resistance to & rho & $p$-value \\
\hline Penicillins (J01C) & Penicillin & 0.57 & 0.008 \\
\hline Macrolides, lincosamides (J01F) & Azithromycin and/or clindamycin & 0.22 & 0.343 \\
\hline \multicolumn{4}{|l|}{ Adult practices } \\
\hline Prescription of & S. aureus resistance to & rho & $p$-value \\
\hline Penicillins (J01C) & Penicillin & 0.30 & 0.319 \\
\hline Macrolides, lincosamides (J01F) & Azithromycin and/or clindamycin & 0.03 & 0.922 \\
\hline \multicolumn{4}{|l|}{ Paediatric practices } \\
\hline Prescription of & S. aureus resistance to & rho & $p$-value \\
\hline Penicillins (J01C) & Penicillin & 0.60 & 0.284 \\
\hline Macrolides, lincosamides (J01F) & Azithromycin and/or clindamycin & 0.54 & 0.391 \\
\hline
\end{tabular}

(Spearman's rank correlation) [significant results marked in bold]

differed between and within the participating countries $[14,16,17]$. Methicillin-resistant S. aureus isolates from patients with impaired health showed increasing fluoroquinolone resistance rate with patients' age. The latter phenomenon was however unique for fluoroquinolones only and was not noticed for other antibiotics [27]. In the healthy population, the resistance rates of different age groups with recent history of increased antibiotic load are likely different from the patients with impaired health $[14,27]$.

The prescribed average volume of all prescribed antibiotics by the participating Hungarian physicians was 15.86 DDD/1000 active patients/day per practice in 2010. The most commonly prescribed antibiotics were the beta-lactam antibacterials, penicillins and the macrolides. According to the data from the European Surveillance of Antimicrobial Consumption (ESAC) project the consumption of antibacterials for systemic use (ATC group J01) was 15.67 DDD/1000 inhabitants/day in the primary care sector in Hungary in 2010, this is comparable to the prescribed average volume of the antibiotics in the cohort of the 20 practices in our study [4].

The nasal carriage of the commensal $S$. aureus and $S$. pneumoniae were higher in the paediatric practices. There were differences between the antimicrobial resistance (resistance to one or more antibiotics - $\mathrm{R} \geq 1$ ) of $S$. aureus and S. pneumoniae in the participating primary care practices, the lowest resistance rates were found in the cohort of the adult practices.

There may be an association between the volume of prescribed antibiotics by primary care physicians and the bacterial resistance rates, the results of the Spearman's rank correlation analysis suggested, that the primary care providers' antibiotic prescribing may correlate with the antibiotic resistance of the commensal S. aureus and S. pneumoniae.

Table 4 Correlations between volume of prescribed penicillins and clarithromycin and/or clindamycin and resistance rates (\%) in all practices, adult and paediatric practices

\begin{tabular}{|c|c|c|c|}
\hline \multicolumn{4}{|l|}{ All practices } \\
\hline Prescription of & S. pneumoniae resistance to & rho & $p$-value \\
\hline Penicillins (J01C) & Penicillin & 0.39 & 0.084 \\
\hline Macrolides, lincosamides (J01F) & Clarithromycin and/or clindamycin & 0.76 & 0.001 \\
\hline \multicolumn{4}{|l|}{ Adult practices } \\
\hline Prescription of & S. pneumoniae resistance to & rho & p-value \\
\hline Penicillins (J01C) & Penicillin & 0.30 & 0.319 \\
\hline Macrolides, lincosamides (J01F) & Clarithromycin and/or clindamycin & 0.63 & 0.021 \\
\hline \multicolumn{4}{|l|}{ Paediatric practices } \\
\hline Prescription of & S. pneumoniae resistance to & rho & $p$-value \\
\hline Penicillins (J01C) & Penicillin & 0.10 & 0.872 \\
\hline Macrolides, lincosamides (J01F) & Clarithromycin and/or clindamycin & 0.52 & 0.191 \\
\hline
\end{tabular}

(Spearman's rank correlation) [significant results marked in bold] 


\section{Strengths and limitations}

There was large amount of information collected. The average volume of the antibiotic prescribing by the participating practices in our study was similar to the previously published Hungarian outpatient antibiotic prescribing data, so the volume of antibiotic prescribing in the participating practices was likely representative for the Hungarian practices $[4,19,20]$. Other strength of the study was the large sample size of the questionnaires and nasal swabs taken from a healthy population. The prevalence and resistance rates were similar to previously published data, but most studies investigated the resistance rates from the hospital settings or from pathogen bacteria $[3,8,12]$. There was no previous study found which investigated the correlation between the antibiotic prescribing of the primary care providers at practice level and the antibiotic resistance of the commensal flora. Previous publication from the APRES Study suggested, that AMR could be associated with the antibiotic exposure in the primary care, it is important to investigate the correlation between the GP practices antibiotic prescribing and the prevalence and resistance of the commensal bacteria on a national level, as we done in our publication [17].

A limitation of the study was the relatively small number of the participating patients and practices, comparing to the total number of primary care practices and to the total population of Hungary, even though the expected number of the practices and participants per country were analysed, calculated and established by an international group of experts and included in the study protocol. The sample was selected as a likely representative sample to the Hungarian primary care practices [13]. The number of the incorrect sampling and the mismatched samples caused some loss of the prevalence and resistance data. The low prevalence and antibacterial resistance of the commensal $S$. pneumoniae made the statistical analyses less powerful. The correlations in our study does not necessarily imply causality.

The antimicrobial resistance can be affected by several factors, some of them cannot be influenced (eg. age of the patient), but there are factors, like the primary care providers' antibiotic prescribing, that can be influenced by education, regulation of the prescribing, developing and use of guidelines etc.

Antibiotic prescribing in the primary care practices may influence the resistance of the commensal flora, which could be a reservoir of resistance genes. According to previous studies, this resistance likely can be transferred from the commensal flora to the pathogens $[10,11]$. Primary care providers prescribe large proportions of the antibiotics; therefore, they have an important role to prevent the spread of the antibiotic resistance. It is very important that the healthcare providers only should prescribe antibiotics only when it's necessary and when the antibiotic treatment is indicated. This can be achieved with appropriate health policies, guidelines and continuous education of the primary care physicians.

\section{Conclusions}

The age is an important influencing factor of antimicrobial resistance. The results suggest that there may be an association between the antibiotic prescribing of the primary care providers and the antibiotic resistance of the commensal S. aureus and S. pneumoniae. The role of the primary care physicians in the appropriate prescribing is very important to avoid the antimicrobial resistance.

\section{Abbreviations \\ ABR: Antibacterial resistance; AMR: Antimicrobial resistance; APRES: The Appropriateness of PREScribing antibiotics in primary health care in Europe with respect to antibiotic resistance; DDD: Defined Daily Dose; DPP: Dose per package; ESAC: European Surveillance of Antimicrobial Consumption; EUCAST: The European Committee on Antimicrobial Susceptibility Testing; GP: General Practitioner; MRSA: Methicillin-resistant Staphylococcus aureus; PC: Primary Care; WHO: World Health Organization}

\section{Acknowledgements}

We would like to thank the contribution of the APRES Study team, with a special thanks to the leaders of the APRES project. We also thank the participating patients, primary care providers, laboratory and administrative staff.

\section{Funding}

The APRES project was funded by the European Commission within the 7th Framework Program (Grant Agreement number 223083). The funding body had no role in the study design and collection, analysis, and interpretation of data or in writing the manuscript.

\section{Availability of data and materials \\ The datasets used and/or analysed during the current study are available from the corresponding author on reasonable request.}

\section{Authors' contributions}

Acquisition of data: LRK, SH, ZJ, GJS. Statistical analysis: JS, GJS. Design, conception, interpretation of data, text writing and revision: LRK, JK, JP, FGS, JS, GJS, SH, ZJ, IR. All authors read and approved the final manuscript.

Ethics approval and consent to participate

The APRES study have been performed in accordance with the Declaration of Helsinki. Ethical permission was authorised for the Hungarian part of the APRES study by the Hungarian Medical Research Councils Scientific and Research Ethics Committees as well (TUKEB-ETT number of permission: 5635-0/2010/1018EKU (401/PI/010). Informed written consent to participate in the study was obtained from all participants or from their parent/guardian in the case of children under 16 years of age.

Consent for publication

"Not applicable".

\section{Competing interests}

The authors declare that they have no competing interests.

\section{Publisher's Note}

Springer Nature remains neutral with regard to jurisdictional claims in published maps and institutional affiliations.

\section{Author details}

'Department of Family and Occupational Medicine, Faculty of Public Health, University of Debrecen, Debrecen, Hungary. ${ }^{2}$ Department of Medical Microbiology, Faculty of Medicine, University of Debrecen, Debrecen, Hungary. ${ }^{3} \mathrm{NIVEL}$, The Netherlands Institute for Health Services Research, 
Utrecht, The Netherlands. ${ }^{4}$ Department of Preventive Medicine, Faculty of Public Health, University of Debrecen, Debrecen, Hungary. ${ }^{5}$ Department of Health Systems Management, Faculty of Public Health, University of Debrecen, Debrecen, Hungary. ${ }^{6}$ Department of General Practice \& Elderly Care Medicine, Amsterdam Public Health Research Institute, VU University Medical Center, Amsterdam, the Netherlands.

Received: 26 June 2018 Accepted: 7 March 2019

Published online: 13 March 2019

\section{References}

1. World Health Organization. Antimicrobial resistance: global report on surveillance. 2014. http://apps.who.int/iris/bitstream/10665/112642/1/ 9789241564748_eng.pdf. Accessed 12 March 2017.

2. Bettiol EO, Harbarth SJ. Development of new antibiotics: taking off finally? Swiss Med Wkly. 2015;145:w14167.

3. Goossens H, Ferech M, Vander Stichele R, Elseviers M. ESAC project group. Outpatient antibiotic use in Europe and association with resistance: a crossnational database study. Lancet. 2005;365:579-87.

4. ESAC-Net interactive database. Country overview of antimicrobial consumption. European Centre for Disease Prevention and Control. http:// ecdc.europa.eu/en/healthtopics/antimicrobial-resistance-and-consumption/ antimicrobial-consumption/esac-net-database/Pages/overview-countryconsumption.aspx. Cited 14 March 2017.

5. Llor C, Bjerrum L. Antimicrobial resistance: risk associated with antibiotic overuse and initiatives to reduce the problem. Ther Adv Drug Saf. 2014;5: 229-41

6. Steinke D, Davey P. Association between antibiotic resistance and community prescribing: a critical review of bias and confounding in published studies. Clin Infect Dis. 2001;33(Suppl 3):S193-205.

7. Hay AD, Thomas M, Montgomery A, Wetherell M, Lovering A, McNulty C, et al. The relationship between primary care antibiotic prescribing and bacterial resistance in adults in the community: a controlled observational study using individual patient data. J Antimicrob Chemother. 2005;56(1):146-53.

8. Costelloe C, Metcalfe C, Lovering A, Mant D, Hay AD. Effect of antibiotic prescribing in primary care on antimicrobial resistance in individual patients: systematic review and meta-analysis. BMJ. 2010 https://doi.org/10.1136/bmj.c2096.

9. Bell BG, Schellevis F, Stobberingh E, Goossens H, Pringle M. A systematic review and meta-analysis of the effects of antibiotic consumption on antibiotic resistance. BMC Infect Dis. 2014;9:13.

10. Levy SB. Factors impacting on the problem of antibiotic resistance. Antimicrob Chemother. 2002;49:25-30.

11. Marshall BM, Ochieng DJ, Levy SB. Commensals: underappreciated reservoir of antibiotic resistance. Microbe. 2009;4:231-8.

12. Andremont A. Commensal flora may play key role in spreading antibiotic resistance. ASM News. 2003;69:601-7.

13. van Bijnen EM, den Heijer CD, Paget WJ, Stobberingh EE, Verheij RA, Bruggeman CA, et al. The appropriateness of prescribing antibiotics in the community in Europe: study design. BMC Infect Dis. 2011. https://doi.org/10. 1186/1471-2334-11-293.

14. den Heijer CD, van Bijnen EM, Paget WJ, Pringle M, Goossens H, Bruggeman CA, et al. Prevalence and resistance of commensal Staphylococcus aureus, including meticillin-resistant Staphylococcus aureus: a European crosssectional study. Lancet Infect Dis. 2013. https://doi.org/10.1016/S14733099(13)70036-7.

15. Boada A, Almeda J, Grenzner E, Pons-Vigués M, Morros R, Juvé R, et al. Prevalencia de portadores nasales de Staphylococcus aureus y Streptococcus pneumoniae en atención primaria y factores asociados a la colonización. Enferm Infecc Microbiol Clin. 2014;33:451-7.

16. Hoffmann $K$, den Heijer CD, George A, Apfalter P, Maier M, et al. Prevalence and resistance patterns of commensal $S$. Aureus in community-dwelling GP patients and socio-demographic associations. A cross-sectional study in the framework of the APRES-project in Austria. BMC Infect Dis. 2015;15:213.

17. van Bijnen EM, Paget J, de Lange-de Klerk ES, den Heijer CD, Versporten A, Stobberingh A, et al. Antibiotic exposure and other risk factors for antimicrobial resistance in nasal commensal Staphylococcus aureus: an ecological study in 8 European countries. PLoS One. 2015;10:e0135094.

18. Matuz M, Benko R, Doro P, Hajdu E, Nagy G, Nagy E, et al. Regional variations in community consumption of antibiotics in Hungary, 1996-2003. Br J Clin Pharmacol. 2006;61:96-100.
19. Benkő R, Matuz M, Viola R, Doro P, Hajdu E, Soós G. Quantitative disparities in outpatient antibiotic exposure in a Hungarian county. J Antimicrob Chemother. 2008;62:1448-50.

20. Matuz M, Benkő R, Hajdú E, Viola R, Soós G. Evaluation of ambulatory antibiotic use in Hungary using drug-specific quality indicators. Orv Hetil. 2013;154:947-56.

21. Rurik I. Primary Care in Hungary. European Forum for Primary Care. http://www. euprimarycare.org/column/primary-care-hungary. Accessed 16 March 2017.

22. European Committee on Antimicrobial Susceptibility Testing. EUCAST definitive document E.DEF. 3.1, June 2000: determination of minimum inhibitory concentrations (MICs) of antibacterial agents by agar dilution. Clin Microbiol Infect. 2000;6:509-15

23. WHO. ATC/DDD Index. World Health Organization. https://www.whocc.no/ atc_ddd_index/. Accessed 22 February 2017.

24. Coenen S, Gielen B, Blommaert A, Beutels P, Hens N, Goossens H. Appropriate international measures for outpatient antibiotic prescribing and consumption: recommendations from a national data comparison of different measures. J Antimicrob Chemother. 2015;69:529-34.

25. Čižman M. Measurement units for antibiotic consumption in outpatients. J Antimicrob Chemother. 2014;69:2877-8.

26. Costelloe C, Lovering A, Montgomery A, Lewis D, McNulty C, Hay AD. Effect of antibiotic prescribing in primary care on meticillin-resistant Staphylococcus aureus carriage in community-resident adults: a controlled observational study. Int J Antimicrob Agents. 2012;39:135-41.

27. Garcia A, Delorme T, Nasr P. Patient age as a factor of antibiotic resistance in methicillin-resistant Staphylococcus aureus. J Med Microbiol. 2017;66(12):1782-9.

\section{Ready to submit your research? Choose BMC and benefit from:}

- fast, convenient online submission

- thorough peer review by experienced researchers in your field

- rapid publication on acceptance

- support for research data, including large and complex data types

- gold Open Access which fosters wider collaboration and increased citations

- maximum visibility for your research: over $100 \mathrm{M}$ website views per year

At BMC, research is always in progress.

Learn more biomedcentral.com/submissions 